\title{
The use of the radial styloid in the extended osteocutaneous radial forearm free flap
}

\author{
Jennifer Matthews MD MSc${ }^{1}$, Wendy $\mathrm{Ng} \mathrm{MD}^{2}$, Stuart Archibald MD³, Carolyn Levis MD MSc ${ }^{4}$
}

\begin{abstract}
J Matthews, W Ng, S Archibald, C Levis. The use of the radial styloid in the extended osteocutaneous radial forearm free flap. Plast Surg 2016;24(2):89-95.
\end{abstract}

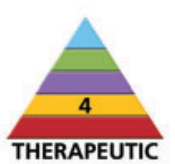

BACKGROUND: The osteocutaneous radial forearm free flap (OC-RFFF) remains a useful tool in head and neck reconstruction; however, it can be challenging to harvest sufficient bone for large reconstructions. The extended OC-RFFF is a modification that involves harvest of the distal border of the radius to the tip of the styloid. This increases the length of the bone flap by $2 \mathrm{~cm}$ to $3 \mathrm{~cm}$, and the inherent contour of the styloid can be used to reconstruct the anterior curvature of the mandible or maxilla without additional osteotomies that may reduce blood supply.

METHODS: The key steps in harvesting the extended OC-RFFF are described. Six patients with mandibular or maxillary defects underwent reconstruction with the extended OC-RFFF. Patient data including demographics, defect features, reconstruction details and outcomes were collected.

RESULTS: Of the six patients who underwent the extended OC-RFFF, four had mandibular reconstruction and two had maxillary reconstruction. Two patients underwent reconstruction for osteoradionecrosis and the remainder for malignant disease. The average patient age at the time of surgery was 64.5 years. The length of radius harvested ranged from $8 \mathrm{~cm}$ to $14 \mathrm{~cm}$ (mean $9.7 \mathrm{~cm}$ ). Two of six patients required a single osteotomy of their bone flap. Donor site complications included partial skin graft loss in $50 \%$ and a radius fracture in two patients. Recipient site complications included one patient with intraoral plate exposure. There have been no cases of nonunion.

CONCLUSIONS: The extended OC-RFFF was a safe and reliable option for bony reconstruction in the head and neck.

Key Words: Head and neck reconstruction; Microsurgery; Osteocutaneous radial forearm free flap

$\mathrm{M}$ andibular and maxillary reconstruction may be required in the setting of malignancy, osteoradionecrosis or trauma. Free tissue transfer using the fibula, radius, scapula, and iliac crest have been used to obtain stable bony and soft tissue reconstruction and recreate functional anatomical relationships (1).

The osteocutaneous radial forearm free flap (OC-RFFF) has remained an extremely usefully tool in head and neck reconstruction $(2-5)$. The enthusiasm for this flap has vacillated due to concern for pathological radius fractures and substandard bone stock relative to other bone flaps such as the fibula (4). With increased use of prophylactic plating of the radius (6) and evidence that functional outcomes (postoperative diet, dentition and trismus), are similar to those using the fibula following mandibular reconstruction $(7,8)$, widespread use of this flap in facial reconstruction has continued $(3,5,9)$.

The advantages of the OC-RFFF include thin, pliable soft tissue, which is ideal for reconstruction of the intraoral lining as well as the reliable skin paddle, long vascular pedicle and ease of harvest (1).

\section{L'utilisation de la styloïde radiale pour le lambeau libre ostéocutané antébrachial radial étendu}

HISTORIQUE : Le lambeau libre ostéocutané antébrachial radial (LL-OAR) étendu demeure utile pour la reconstruction de la tête et du cou. Cependant, il peut être difficile de prélever assez d'os pour effectuer des reconstructions importantes. Le LL-OAR étendu est une modification qui consiste à lever le bord distal du radius à la pointe de la styloïde. Cette technique accroît la longueur du lambeau osseux de $2 \mathrm{~cm}$ à $3 \mathrm{~cm}$ et permet d'utiliser le contour inhérent de la styloïde pour reconstruire la courbure antérieure de la mandibule ou de la maxillaire sans ostéotomies supplémentaires susceptibles de réduire l'apport sanguin.

MÉTHODOLOGIE : Les auteurs ont décrit les principales étapes pour lever le LL-OAR étendu. Six patients présentant des anomalies mandibulaires ou maxillaires ont subi une reconstruction par LL-OAR étendu. Les auteurs ont décrit les données sur les patients, y compris les données démographiques, les caractéristiques des anomalies, les détails et les résultats de la reconstruction.

RÉSULTATS : Des six patients qui ont reçu un LL-OAR étendu, quatre ont subi une reconstruction mandibulaire et deux, une reconstruction maxillaire. Dans deux cas, la reconstruction était causée par une ostéoradionécrose et dans les autres, par un cancer. Les patients avaient un âge moyen de 64,5 ans au moment de l'opération. Le radius prélevé était d'une longueur de $8 \mathrm{~cm}$ à $14 \mathrm{~cm}$ (moyenne de $9,7 \mathrm{~cm}$ ). Deux des six patients ont dû subir une seule ostéotomie de lambeau osseux. Les complications au foyer du donneur incluaient une perte partielle de la greffe cutanée dans $50 \%$ des cas et une fracture du radius chez deux patients. Les complications au foyer du receveur incluaient un patient dont la plaque intra-orale avait été exposée. Il n'y a eu aucun cas de non-union.

CONCLUSIONS : Le LL-OAR étendu était sécuritaire et fiable pour procéder à la reconstruction osseuse de la tête et du cou.

Commonly cited limitations of the OC-RFFF are the length and thickness of bone available and the limited number of osteotomies that can be safely preformed $(1,4)$. Traditionally, the maximum length of bone that can be harvested is approximately $8 \mathrm{~cm}$ to $12 \mathrm{~cm}$, and lies between the insertions of the brachioradialis (BR) and pronator teres $(3,10,11)$. Although multiple osteotomies are technically possible, the best way to preserve bone perfusion is to limit the number of osteotomies. Consequently, in patients requiring a long segment of bone, the fibula flap is often selected for reconstruction (1). Faced with challenging patients, in whom the fibula is not desirable due to significant peripheral vascular disease or congenital variations in vascular anatomy, we modified the OC-RFFF to safely facilitate larger reconstructions.

We report our experience in six patients in which the OC-RFFF was extended to include the distal border of the radius to the tip of the styloid. The 'extended' OC-RFFF provides access to and extra $2 \mathrm{~cm}$ to $3 \mathrm{~cm}$ of quality bone that is not an essential component of the radius or

${ }^{1}$ Section of Plastic Surgery, University of Calgary, Calgary, Alberta; ${ }^{2}$ Division of Plastic Surgery, McMaster University; ${ }^{3}$ Division of

Otolaryngology Head $\mathbb{E}$ Neck Surgery; St Joseph's Hospital, McMaster University; ${ }^{4}$ Division of Plastic Surgery; St Joseph's Hospital;

McMaster University; Hamilton, Ontario

Correspondence: Dr Jennifer Matthews, Section of Plastic Surgery, University of Calgary, \#200, 2004 14th Street Northwest, Calgary,

Alberta T2M 3N3. Telephone 403-474-0113, fax 403-474-7937, e-mail jennifer.matthews1@me.com 


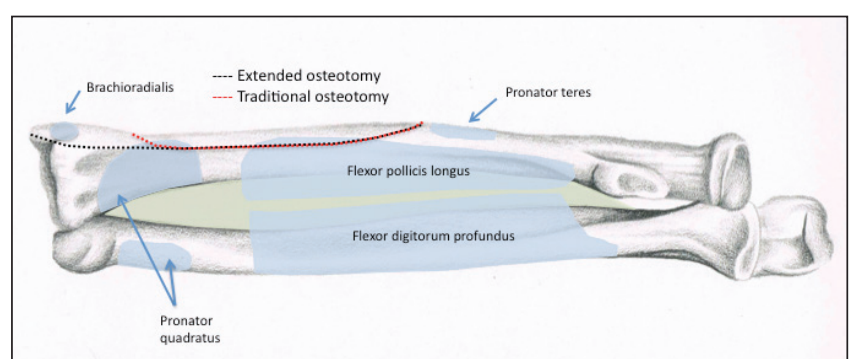

Figure 1) Classical osteotomy for the osteocutaneous radial forearm free flap (OC-RFFF) (red dotted line). Proposed osteotomy for extended OC-RFFF including the radial styloid (black dotted line). Muscular insertions are indicated

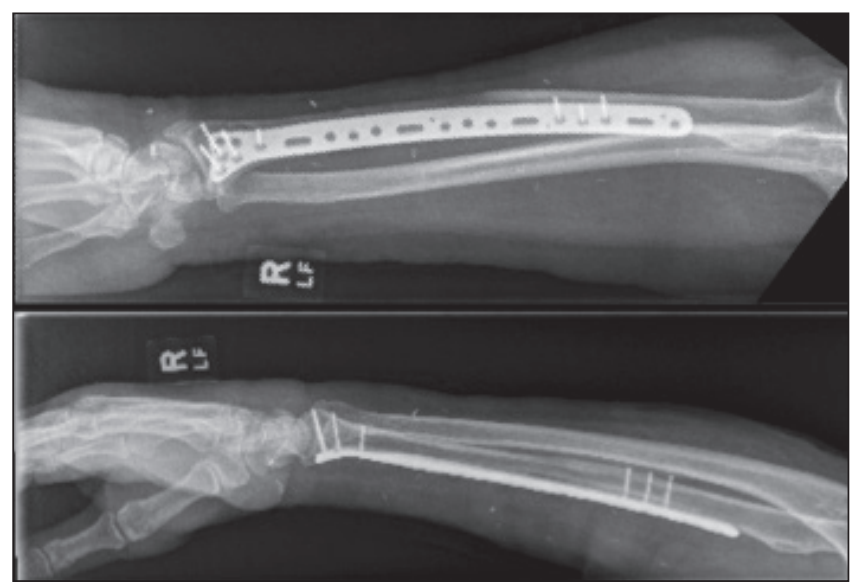

Figure 2) Postoperative $x$-ray revealing prophylactic internal fixation in patient 5

radiocarpal joint (12). The contour of the radial border of the styloid is used specifically to reconstruct the anterior mandible or maxilla, where its inherent shape can recreate the contour without the need for additional osteotomies. This enables reconstruction of longer segments without negatively impacting bone perfusion.

The extended OC-RFFF is raised in the typical fashion with the following modifications: the BR insertion is sharply removed from the radius; the distal border of the radius to the tip of the styloid is exposed in an extracapsular fashion and included in distal osteotomy; the radioscaphocapitate ligament is preserved; the defect in the radius is packed with synthetic bone substitute (DYNA-Graft, USA). A reconstruction plate is applied for any OC-RFFF, including the extended OC-RFFF, if the harvested bone is greater than one-third of the crosssectional area or the patient is considered to be high risk for postoperative fracture of the radius. The curve of the radial border to the tip of the styloid is used specifically to recreate the anterior contour of a mandible or maxilla; this reduces the need for multiple osteotomies. The BR tendon is reinserted via drill holes to the remaining radius to provide maximum coverage of the synthetic bone and the plate.

\section{METHODS}

The present study was a retrospective review of six consecutive patients who underwent mandibular or maxillary reconstruction with the extended OC-RFFF from December 2012 to February 2014. Data were collected through a retrospective chart review. Ethics approval was granted by Hamilton Integrated Research Ethics Board (Hamilton, Ontario) (ID: 14-179-C).

Patients were identified by the senior author (CL). All ablative and reconstructive surgeries were performed by the senior authors (CL, $\mathrm{SA})$ at St Joseph's Hospital (Hamilton, Ontario). Postoperative follow-up ranged from six to 19 months (at the time of the study) and remains active. Data obtained in the chart review included patient age, location and etiology of defect, length of stay in hospital and

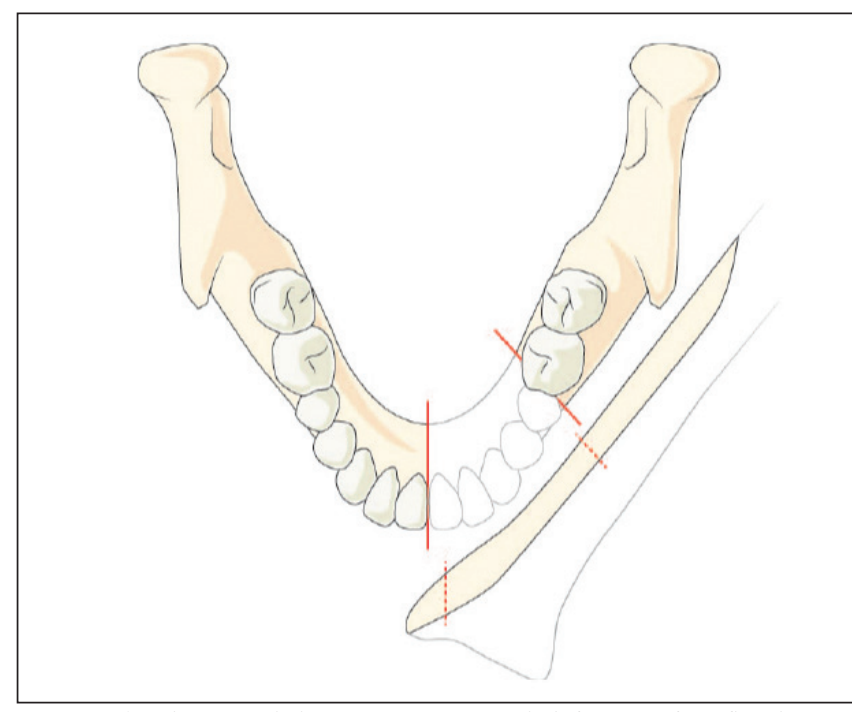

Figure 3) The extended osteocutaneous radial forearm free flap has an inherent curvature that allows reconstruction of anterior-lateral defects without the need for additional osteotomies

complications (systemic, donor site, recipient site). Reconstructive details collected include the length of bone harvested, number of osteotomies, plating of donor radius, location of skin paddle placement and the use of secondary free flaps. Relevant imaging of the facial skeleton and the radius were reviewed to assess bony union and complications. Subjective and objective assessments were conducted to evaluate the morbidity of the flap harvest on the function of the donor arm.

\section{Surgical technique}

The initial harvest of the OC-RFFF is performed as classically described $(1,10)$. Preoperative forearm markings centre the flap volarly over the distal radial artery, with a skin island marked to match the mucosal defect size and shape. After elevation of the standard fasciocutaneous component of the flap under tourniquet control, the radius is exposed. The BR tendon is retracted radially to protect and preserve the periosteal perforators. A scalpel is used to incise the pronator quadratus, flexor pollicis longus and periosteum as far proximally as required to achieve sufficient length and exposure for osteotomies. Finally, the BR tendon is sharply disinserted to gain access to the radial border and styloid tip. The bone now available for harvest stretches from the tip of the radial styloid proximally to, or slightly beyond, the pronator teres insertion (Figure 1). The proposed osteotomy site is marked. Care is taken to include a keel-shaped taper at the proximal and distal ends as described by Weinzweig et al (13). The design of this osteotomy removes a portion of the radial styloid without breaching the wrist capsule and preserves the radioscaphocapitate ligament. A sagittal saw is used to complete the osteotomy with a goal of removing a maximum of one-third of the cross-sectional area of the radius. The flap dissection is completed with pedicle dissection from distal to proximal. The communicating vein between the superficial and deep venous systems is preserved when possible. The tourniquet is released to visualize perfusion of the flap and donor limb before removing the flap for transfer to the head and neck defect.

The bony defect in the radius is packed with a synthetic bone substitute (DynaGraft, USA). The donor radius is plated prophylactically when the ostectomized bone represents more than one-third of the radius thickness in any location or if the patient is considered to be high risk for fracture of the radius (ie, osteoporosis, ambulates using a cane or walker). A reconstructive locking plate is used on the volar surface (VariAx, Stryker, USA). Three bicortical screws are placed proximal to the osteotomy and the plate architecture allows for two to three screws to be placed ulnar to the osteotomy at the styloid in the remaining distal radius (Figure 2). The BR tendon is reinserted into 
TABLE 1

Demographics, comorbidities, reconstructive details and complications involving extended osteocutaneous radial forearm free flap (RFFF) patients $(n=6)$

\begin{tabular}{|c|c|c|c|c|c|c|c|c|c|c|c|c|c|}
\hline \multirow[b]{3}{*}{ Patient } & \multirow{3}{*}{$\begin{array}{c}\text { Age, } \\
\text { years/sex }\end{array}$} & \multirow{3}{*}{$\begin{array}{l}\text { Length } \\
\text { of stay, } \\
\text { days }\end{array}$} & \multirow{3}{*}{$\begin{array}{c}\text { F/U, } \\
\text { months }\end{array}$} & \multirow{3}{*}{$\begin{array}{c}\text { Co- } \\
\text { morbidities }\end{array}$} & \multirow{3}{*}{$\begin{array}{l}\text { Defect } \\
\text { etiology }\end{array}$} & \multirow{3}{*}{$\begin{array}{l}\text { Bone } \\
\text { defect }\end{array}$} & \multirow{3}{*}{$\begin{array}{l}\text { Soft tissue } \\
\text { reconstruction }\end{array}$} & \multirow{3}{*}{$\begin{array}{l}\text { Radius } \\
\text { used, } \mathrm{cm}\end{array}$} & \multirow{3}{*}{$\begin{array}{l}\text { Pre- } \\
\text { plating }\end{array}$} & \multirow{3}{*}{$\begin{array}{l}\text { Osteo- } \\
\text { tomies }\end{array}$} & \multicolumn{3}{|c|}{ Complications } \\
\hline & & & & & & & & & & & \multirow[b]{2}{*}{ Systemic } & \multicolumn{2}{|c|}{ Site } \\
\hline & & & & & & & & & & & & Donor & Recipient \\
\hline \multirow[t]{2}{*}{1} & 64/female & \multirow[t]{2}{*}{30} & \multirow[t]{2}{*}{17} & PVD & ORN & Mandible & 1. Intra-oral & 12 & No & 0 & & 1. Radius & \multirow{2}{*}{$\begin{array}{l}\text { 1. Plate } \\
\text { exposure } \\
\text { (intra-oral) }\end{array}$} \\
\hline & & & & & & & $\begin{array}{l}\text { 2. Extra-oral } \\
\text { with submen- } \\
\text { tal artery FF) }\end{array}$ & & & & & & \\
\hline \multirow{2}{*}{2} & \multirow{2}{*}{ 59/male } & \multirow{2}{*}{7} & \multirow{2}{*}{15} & HTN & \multirow{2}{*}{ ORN } & \multirow{2}{*}{ Mandible } & \multirow{2}{*}{$\begin{array}{l}\text { 1. Intraoral with } \\
\text { RFFF }\end{array}$} & \multirow{2}{*}{8} & \multirow{2}{*}{ Yes } & \multirow{2}{*}{0} & \multirow{2}{*}{\multicolumn{3}{|c|}{$\begin{array}{l}\text { 1. Exposed } \\
\text { tendon }\end{array}$}} \\
\hline & & & & DM-2 & & & & & & & & & \\
\hline \multirow[t]{2}{*}{3} & \multirow[t]{2}{*}{$65 /$ male } & 8 & 13 & HTN & Adeno-CA & Maxilla & $\begin{array}{l}\text { 1. Intraoral with } \\
\text { RFFF }\end{array}$ & 6 & $\mathrm{No}^{*}$ & 0 & & $\begin{array}{l}\text { 1. Radius } \\
\text { fracture }\end{array}$ & \\
\hline & & & & & & & & & & & & $\begin{array}{l}\text { 2. Infection } \\
\text { with par- } \\
\text { tial skin } \\
\text { graft loss }\end{array}$ & \\
\hline & & & & & & & $\begin{array}{l}\text { 2. Extra-oral } \\
\text { with submen- } \\
\text { tal artery FF) }\end{array}$ & & & & & & \\
\hline 5 & $72 /$ male & 47 & 5 & PVD & SCC & Mandible & 1. Intra-oral & 14 & Yes & 1 & Postop MI & 1. Tendon & 1. Partial \\
\hline & & & & HTN & & & with RFFF & & & & & exposure & necrosis \\
\hline & & & & DM-2 & & & 2. Extra-oral & & & & & & of intra- \\
\hline & & & & CAD & & & $\begin{array}{l}\text { with RFFF } \\
\text { (folded) }\end{array}$ & & & & & & $\begin{array}{l}\text { oral skin } \\
\text { island }\end{array}$ \\
\hline 6 & $69 /$ male & 9 & 4 & PVD & $\mathrm{SCC}$ & Mandible & 1. Intraoral with & 10 & No & 0 & & & \\
\hline & & & & HTN & & & RFFF & & & & & & \\
\hline & & & & CAD & & & & & & & & & \\
\hline
\end{tabular}

*Patient 3 underwent over-resection of the proximal volar cortex, which was secured with two lag screws. No plate was placed at the time of osteocutaneous RFFF harvest. Adeno-CA Adencarcinoma; CAD Coronary artery disease; DM-2 Type2 diabetes mellitus; FF Free flap; FU Follow-up; HTN Hypertension; MI Myocardial infarction; ORN Osteoradionecrosis; Postop Postoperative; PVD Peripheral vascular disease; SCC Squamous cell carcinoma

the radius via two parallel drill holes and a 2-0 Ti-Cron suture. The defect is closed in layers with full muscular coverage of the plate. A split thickness skin graft is harvested from the patient's thigh and used to resurface the skin defect. The skin graft is protected with a bolster dressing and the arm is secured in a hand-based above elbow splint until the first dressing change at postoperative day 7 . An above-elbow splint is maintained for three weeks to protect the BR repair. This is followed by below-elbow splinting for an additional three weeks when a large segment of radius is taken.

The flap is inset before revascularization. The distal end of the radius that includes the curve of the styloid is positioned at the most anterior portion of the bone defect to take advantage of inherent curvature (Figure 3). An additional closing wedge osteotomy may be preformed to optimize the contour of the reconstruction. The bone flap is typically secured to the mandibular or maxillary plate that is placed at the time of the ablation to maintain anatomical relationships. The most common location for the skin island is intraoral. One patient (patient 5) had the OC-RFFF skin island folded to also resurface a coexisting extra-oral defect in addition to an intra-oral paddle. All other extra-oral defects were reconstructed with a second flap (submental artery free flap, patients 1 and 4). The radial artery and venous system were anastomosed to vessels in the ipsilateral neck to revascularize the flap. Patients were managed on the Head \& Neck Unit at St Joseph's Hospital. Flap monitoring is clinical and postoperative imaging is performed on an as-needed basis.

Patients are followed by both the ablative and the reconstructive (SA and CL, respectively) surgeons every three to four weeks for the first several months to years.
TABLE 2

Patient comorbidities $(n=6)$

\begin{tabular}{lc}
\hline Comorbidity & $\mathbf{n}(\%)$ \\
\hline Smoker & $6(100)$ \\
Alcohol & $2(33)$ \\
Diabetes & $2(33)$ \\
Hypertension & $5(83)$ \\
Chronic obstructive pulmonary disease & $0(0)$ \\
Coronary artery disease & $2(33)$ \\
Peripheral vascular disease & $4(67)$ \\
\hline
\end{tabular}

\section{RESULTS}

Between December 2012 and February 2014, a total of six patients underwent head and neck reconstruction using the modified OC-RFFF described above (Table 1). Four patients underwent mandibular reconstruction and two had maxillary reconstruction. Two patients had reconstruction for osteoradionecrosis (ORN) and remainder for malignant disease. The average patient age at the time of surgery was 64.5 years (range 58 to 72 years). Patient 1 is female but all subsequent patients are male. The average length of stay in the hospital was 18.5 days (range seven to 47 days).

The presence of selected comorbidities was recorded at the time of the chart review (Table 2). Four patients were active smokers while the remaining two had quit within six months of surgery. All six patients had hypertension, two had diabetes and another two had 

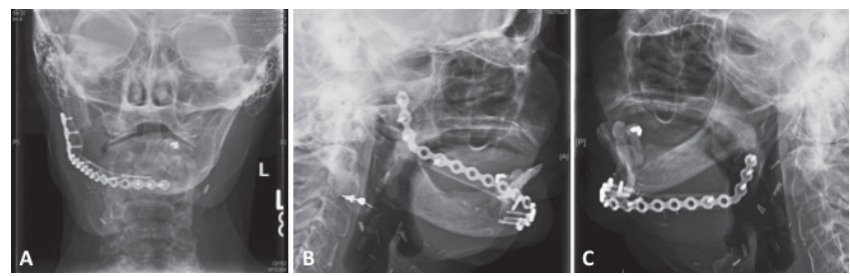

Figure 4) Mandibular reconstruction in Patient 1. A Anterior/posterior view. B Left lateral/oblique. C Right lateral

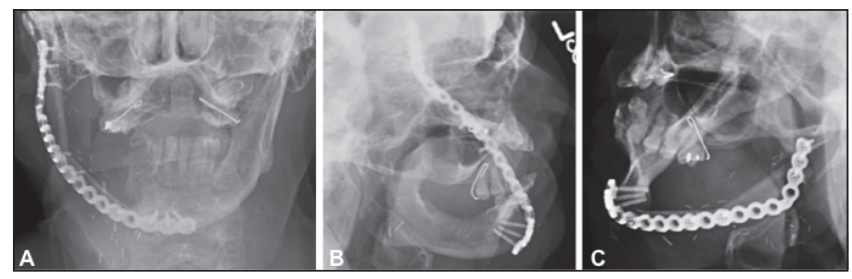

Figure 5) Patient 5 mandibular reconstruction after right sided resection from 4-2 tooth to ascending ramus (inclusive). A Anterior/posterior view. B Left lateral/oblique. C Right lateral

significant coronary artery disease. Four of six patients had peripheral vascular disease on angiography or physical examination suffciently significant to preclude the use of the fibular flap. The other two patients (patients 3 and 4) were reasonable candidates for fibular flaps but required maxillary reconstruction and the OC-RFFF was chosen as the best flap option. Three of six patients required extra-oral soft tissue reconstruction in addition to their intraoral defect. Two patients had a submental artery free flap to reconstruct the extra-oral defect (patients 1 and 4 ) and patient 5 had a folded OC-RFFF to resurface both the intra-oral lining and extra-oral coverage. Four patients did not require osteotomies as part of their reconstruction, the remaining two required a single osteotomy; however, the curve of the distal radial border and tip of styloid was used to restore the necessary contour. The length of radius harvested ranged from $8 \mathrm{~cm}$ to $14 \mathrm{~cm}$ (mean $10 \mathrm{~cm}$ ). Two flaps required a single osteotomy to complete the reconstruction. Bone harvest was limited to an estimated one-third of the cross-sectional area of the radius. Patient 1 required a segmental mandibulectomy from the right lateral incisor to the mandibular angle (inclusive) to treat ORN. Reconstruction was completed with a $12 \mathrm{~cm}$ OC-RFFF and no osteotomies (Figure 4).

Patient 5 required a segmental mandibulectomy from the symphysis (4-2 tooth) to the ascending ramus (inclusive) to treat squamous cell carcinoma. Mandibular reconstruction was performed with an extended OC-RFFF including a $14 \mathrm{~cm}$ long segment of radius (Figure 5). Intraoperative photos demonstrate how the inherent curvature of the radial styloid was used to recreate the contour of the anterior mandibular symphysis (Figure 6). A single osteotomy was needed proximally on the bone flap to recreate the contour of the mandibular body and complete the reconstruction. Patient 5 underwent prophylactic internal fixation (PIF) following flap harvest.

All patients had synthetic bone substitute placed in the ostectomy defect (DynaGRAFT, USA). Three of six patients underwent PIF of the radius. Figure 4 shows patient 5 , who underwent PIF and harvest of a $14 \mathrm{~cm}$ segment of radius. Following reconstruction with a locking plate, three screws were placed proximal and four screws were placed distally to the ostectomy (VariAx plate, Stryker, USA). The decision to plate the radius is influenced by the amount of bone harvested (ie, greater than one-third in any region), and additional patient factors (ie, osteoporosis, require assisted ambulation with cane or walker).

Complications were categorized as systemic, donor site (forearm) or recipient site (head and neck). Patient 5 experienced a myocardial infarction on postoperative day 1 , which required medical intervention only. Multiple comorbidities and a pre-existing wheelchair dependence led to a prolonged hospital stay of 47 days for this patient.
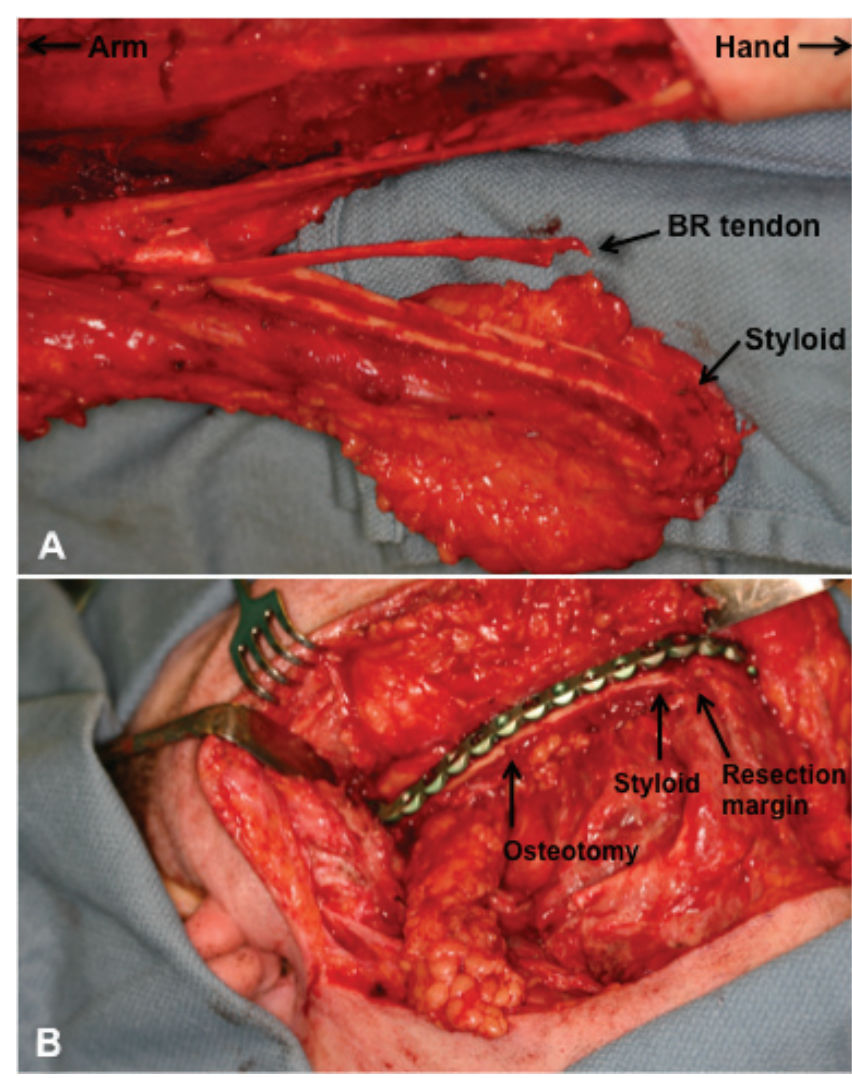

Figure 6) Patient 5 intraoperative photos. A Right forearm with $14 \mathrm{~cm}$ extended osteocutaneous radial forearm free flap intact. Note the inherent curvature of the styloid portion of the bone. B Right neomandible reconstruction from symphysis to ascending ramus. Note the single osteotomy at the body and curvature at the symphysis from the styloid

Recipient site complications were minimal; there were no flap failures. All patients had clinical evidence of bony union based on the absence of symptoms at the site of bone coaptation on physical examination. Patient 1 had exposure of the mandibular reconstruction plate that required local debridement and removal of a small segment of the plate. Because there was bony union, the wound was primarily closed and the patient experienced no further issues.

Patient 5 experienced partial necrosis of the intra-oral skin paddle. The forearm flap was folded to facilitate reconstruction of both intraoral and extra-oral wounds. The intervening bridge of tissue was deepithelialized, which may have affected perfusion in this thin flap. The wound healed with debridement and local wound care. The remainder of the intra-oral soft tissue and the extra-oral component of the flap were viable in this patient. Recipient site complications included partial skin graft loss in three $(50 \%)$ patients. There was a donor site infection in one of these patients. All donor sites healed by secondary intention with wound care and dressings.

Two patients had fractures of their donor radius (patients 1 and 4). In both cases, the fractures occurred in the typical location following a OC-RFFF (central one-third of the radius) and, therefore, are not believed to be related to harvesting the distal border of the radial styloid. Neither patient underwent PIF, and both were identified to have had inadvertent over-resection and/or risk factors for poor bone health/healing. Both patients were treated with open reduction and internal fixation (ORIF) supplemented by synthetic or autologous bone graft.

Functional outcomes, such as postoperative diet, dentition and trismus, were not a focus of the present chart review given the relatively limited follow-up period (range six to 19 months). Based on standard physical examinations, elbow flexion, pronation and 
supination have not been impacted by BR removal and reinsertion or by postoperative immboilization. Ulnar translocation was not present on postoperative wrist $\mathrm{x}$-rays and there were no plating complications. All mandibular reconstructions are currently stable. At the time of chart review, all patients were alive; four patients remain disease free. Patient 2 presented again with metastatic disease six months after undergoing reconstruction for ORN, and patient 4 presented with a local recurrence two months after the initial ablative and reconstructive procedure. All patients are tolerating an oral diet, although the most recent patient in the study is continuing to supplement with gastrostomy tube feeds at three months postoperatively. None of the patients have pursued dental rehabilitation with osteointegrated implants and, although it is believed that radius bone is less optimal for implants, the cited reason is prohibitive cost for these patients.

\section{DISCUSSION}

The current study describes a novel modification of the OC-RFFF that extends the osteotomy distally to maximize bone quantity and quality. We believe this modification could be useful to any surgeon performing head and neck reconstruction for defects related to trauma, cancer or ORN. For surgeons who already use the OC-RFFF as their preferred flap, this provides a technique to increase the length and quality of radius harvested. The natural curvature of the radial styloid may also preserve bone perfusion by reducing the number of osteotomies needed for an anatomical reconstruction. For surgeons who use the fibula or other flap as their first choice in bony reconstruction, this flap provides an alternative when their fist choice is not suitable. The presence of peripheral vascular disease, congenital variants of vessels in the leg or concerns about immediate and long-term ambulation may remove the fibula flap as an option $(1,14)$. The patient population represented in the present study was challenging and necessitated creative reconstructive solutions.

Without the limitation of the BR insertion, we were able to include naturally contoured bone from the styloid, lengthen the bone flap by $2 \mathrm{~cm}$ to $3.5 \mathrm{~cm}$ and reconstruct large defects while limiting osteotomies. The radial styloid consists of thick cortical bone, and is commonly removed to treat wrist arthritis and does not negatively impact wrist biomechanics when the volar radioscaphocapitate ligament is preserved (12). In our patients, it is the radial border to the tip of the styloid that is harvested and, therefore, less bone is resected than for a styloidectomy. The curve of the styloid, when placed at the anterior border of the mandible or maxilla, eliminates the need for this osteotomy (Figure 3). Two patients described in the present report required a single osteotomy. A lateral osteotomy was required in patient 5 for a hemimandibular defect from the symphysis (lateral incisor) to the ascending ramus. The contour of the radial styloid eliminates the need for the anterior, osteotomy; however, the large defect required an osteotomy to recreate the mandibular angle. Patient 4 had a complex maxillary defect that necessitated a lateral osteotomy to reach the lateral buttress and conform to the reconstruction plate.

Due to its wide use, the OC-RFFF is extensively studied $(2,3,7,8,15)$. These studies show that bony reconstruction with the radius is safe and effective. Arganbright et al (3), and Sinclair et al (9), recently published a large, complication-focused, series of 167 and 218 consecutive patients, respectively. All patients had head and neck reconstruction using the OC-RFFF. The rates of recipient site infection, bone or plate fracture, malunion/nonunion were found to be very low ( $1 \%$ to $3 \%$ each) (3). Donor site complications were similarly low, including a single radius fracture in each series (incidence $0.45 \%$ to $0.6 \%)(3,9)$. Notably, recipient site hardware exposure was $16 \%$ to $17 \%$, a value that is higher than typically reported for the fibula flap $(16,17)$, but consistently reported for the OC-RFFF $(9,15)$. This complication occurred in one of the six patients $(16.7 \%)$ in the present study. Dean et al (7) and Virgin et al (8) have both compared the OC-RFFF to the fibula. The results show that these flaps are similar with respect to complications and provide equivalent functional outcomes $(7,8)$. Interestingly, despite evidence suggesting the OC-RFFF cannot support dental implants (18), both studies reported more patients in the OC-RFFF group receiving implants than in the fibula group $(7,8)$. Dental implants typically require the addition of supplemental bone graft in the case of the OC-RFFF or fubula. As a result of these long-term outcome studies, we are confident in recommending the OC-RFFF, including the modification described here. We hypothesize that the bone flap described here would help improve recipient site outcomes because of increased bone stock at the styloid and the need for fewer osteotomies. Patient 1 is the only patient to experience significant recipient site (intra-oral) morbidity with plate exposure requiring reoperation. Nevertheless, the bone flap was united, allowing for the segment of exposed plate to be removed and primary closure of the soft tissue. Patient 5 experienced partial necrosis of the flap soft tissue, requiring debridement and wound care only. A subsequent study with more patients, longer follow-up and comparison of the modified OC-RFFF with traditional OC-RFFF and fibular reconstructions in our own patient population is necessary.

For this modification to be successful, it important that there is no additional donor site morbidity. A major deterrent to the use of the OC-RFFF is the risk of a pathological radius fracture. A fracture can be a significant setback for patients because it may delay recovery and rehabilitation. Operative repair is often not straightforward given the presence of skin grafts, scar and a bony defect requiring bone graft $(19,20)$.

Early reports have a $15 \%$ to $30 \%$ incidence of radius fractures $(19,21,22)$. Subsequently, biomechanical studies demonstrated the benefit of PIF (6), limiting the osteotomy to $30 \%$ of the circumference (23), and bevelling at the proximal and distal ends $(13,23,24)$. Further refinements in the setting of PIF include using a low-contact dynamic compression plate (25), excluding screws from the osteotomized portion of the radius (25) and adding bone graft to the donor radius (11). The rate of fracture when all patients undergo PIF was $0.5 \%$ in two large retrospective reviews $(3,9)$, and $0 \%(25,26)$ to $9.6 \%$ in smaller similar series $(5,27)$. Routine PIF appears to have reduced the fracture risk associated with the OC-RFFF, even in the setting of large bone flaps (harvest of $40 \%$ to $50 \%$ of the radius circumference) $(3,7,25)$. Because this is a new modification, there are no data regarding the effect of including the border of the radial styloid on fracture risk, although we suspect it will not affect the fracture rate because invariably, the fracture occurs proximally where the radius is the narrowest. Radial styloidectomy is used commonly in the treatment of wrist arthritis and is considered to be a safe procedure with few complications (28). The current literature does not contain any case reports describing fractures following radial styloidectomy. A single in vitro study demonstrated that excess radial styloidectomy may affect wrist stability but that small resections, similar what is done in the extended OC-RFFF are safe (12).

Two of six $(33 \%)$ patients in our series had a radius fracture. In both cases, the fracture occurred in the location most common after an OC-RFFF: the central one-third at the proximal osteotomy. In both cases, there appears to have been inadvertent over-resection of the radius, placing them at risk for a fracture. Patient factors for poor health and healing must also be considered.

Patient 1 was found to have a pathological right radius fracture less than one month after surgery after lifting a book. She had a $12 \mathrm{~cm}$ segment of bone harvested to reconstruct a large hemimandibular defect secondary to osteoradionecrosis that required two flaps to resurface intra- and extra-oral defects (OC-RFFF and submental artery flap) (Figure 4). Although she was noted to have a narrow radius, she did not undergo PIF becuase it was believed that $<30 \%$ of the radius had been harvested. In hindsight, there was likely over-resection of her radius. This patient was not an ideal candidate for a fibular flap and had other signs of poor bone health. She had a history of nonhealing leg ulcers bilaterally, nonpalpable pulses on the right leg, poor pulses and an un-united patella fracture the left leg. Furthermore, she also had a history of a fracture of the contralateral forearm six months before the OC-RFFF. Following ORIF of her fracture, she went on to 
experience exposure of her mandibular hardware requiring further surgery to remove a portion of the plate. There was bony union of her neomandible and has fared fairly well, other than a hip fracture $<1$ year after the head and neck reconstruction.

Patient 3 was found to have a fractured radius approximately five weeks postoperatively. Although he did not have an overly long or wide segment of bone harvested $(6 \mathrm{~cm}$ with $30 \%$ cross-sectional area of the radius), he had over-resection of the volar cortex that was noted intraoperatively. Despite placing lag screws to secure this volar segment, the radius ultimately had a fracture. It is possible that the placement of the lag screws created a stress riser because this is where the fracture occurred. There is some evidence that the specific type of plate used for PIF can modify the risk for fracture (4); however, we are not aware of any studies investigating lag screws. The fracture occurred in the location that is typical following the harvest of an OC-RFFF and we do not believe the specific technique of harvesting an extended flap contributed to the fracture. This patient had a good outcome after ORIF and bone grafting.

It is difficult to determine whether PIF would have prevented fractures in these patients. The fracture risk without PIF is not $100 \%$ $(19,21,22)$ and prophylactic plating has not eliminated fractures $(3,10)$. Studies have indicated that harvesting bone from the radius significantly impacts the strength of the bone $(23,24)$. The diameter and length of bone removed appear to have an incremental impact on strength (24). Our technique facilitates a longer bone flap and, therefore, could theoretically increase the risk for a pathological fracture; however, the length is added distally, where fractures do not occur. To date, there is insufficient evidence to determine the exact risk associated with the length of radius harvested, and long bone segments (up to $12 \mathrm{~cm}$ ) are being routinely harvested by other surgeons $(3,5,25)$.

Patient comorbidities and other patient factors likely have a significant impact on the risk for complications including pathological radius fractures. Head and neck oncology patients often have risk factors associated with poor healing including a history of smoking, diabetes, poor nutrition and vitamin/mineral deficiencies, peripheral vascular disease and local radiation therapy. Osteoporosis and osteopenia also share many risk factors with this patient population including older age, smoking/chronic obstructive pulmonary disease,

\section{REFERENCES}

1. Microsurgical Rconstruction of the Head and Neck. St Louis: Quality Medical Publishing, Inc; 2010.

2. Andrades P, Rosenthal EL, Carroll WR, Baranano CF, Peters GE. Zygomatic-maxillary buttress reconstruction of midface defects with the osteocutaneous radial forearm free flap. Head Neck 2008;30:1295-302.

3. Arganbright JM, Tsue TT, Girod DA, et al. Outcomes of the osteocutaneous radial forearm free flap for mandibular reconstruction. JAMA Otolaryngol Head Neck Surg 2013;139:168-72.

4. Avery CM. Review of the radial free flap: Is it still evolving, or is it facing extinction? Part one: Soft-tissue radial flap. Br J Oral Maxillofac Surg 2010;48:245-52.

5. Kim JH, Rosenthal EL, Ellis T, Wax MK. Radial forearm osteocutaneous free flap in maxillofacial and oromandibular reconstructions. Laryngoscope 2005;115:1697-701.

6. Bowers KW, Edmonds JL, Girod DA, Jayaraman G, Chua CP, Toby EB. Osteocutaneous radial forearm free flaps. The necessity of internal fixation of the donor-site defect to prevent pathological fracture. J Bone Joint Surg Am 2000;82:694-704.

7. Dean NR, Wax MK, Virgin FW, Magnuson JS, Carroll WR, Rosenthal EL. Free flap reconstruction of lateral mandibular defects: Indications and outcomes. Otolaryngol Head Neck Surg 2012;146:547-52.

8. Virgin FW, Iseli TA, Iseli CE, et al. Functional outcomes of fibula and osteocutaneous forearm free flap reconstruction for segmental mandibular defects. Laryngoscope 2010;120(Suppl 4):S190.

9. Sinclair CF, Gleysteen JP, Zimmermann TM, et al. Assessment of donor site morbidity for free radial forearm osteocutaneous flaps. Microsurgery 2012;32:255-60. excess alcohol intake, lack of exercise and calcium/vitamin D deficiency (29). Patient 1 was an active smoker with a history of fractures, including an un-united patella fracture at the time of surgery, and comorbidities including significant peripheral vascular disease and hypertension. Conversely, patient 3 was relatively healthy without an obvious fracture risk.

In most circumstances, the decision to add PIF is made by the reconstructive surgeon following bone flap removal. Placing a plate on the donor radius is not simple or risk free (4). It adds time, complexity and cost to a procedure, which inherently consume a great deal of resources and this may not be cost effective (30). However, when a fracture occurs with fixation in place, treatment is less difficult because the fractures tend not to displace $(4,5,10,27)$. Taking this information into consideration, there should be a low threshold to perform PIF in patients undergoing an OC-RFFF. The patient's health, comorbidities, fracture risk and lifestyle factors (ie, requires assisted ambulation with cane/walker) should all be considered in addition to the amount of bone harvested.

\section{CONCLUSION}

The technique of the extended radial forearm osteocutaneous flap with inclusion of the distal radial border to the tip of the styloid can provide bony length and contour and is a viable modification of the standard operation. This modification can be considered when approaching free flap reconstruction of mandibular and maxillary defects in which the contour of the radial styloid can help recreate the anterior curvature without an osteotomy and without impacting perfusion to the bone flap. We believe this is a safe and effective flap; however, a study with more patients, longer follow-up and a comparison with the traditional OC-RFFF is required. There should be a low threshold for PIF, particularly in the head and neck oncology population, who may have a higher fracture risk based on their comorbidity profiles or require assisted ambulation (ie, walker or cane).

DISCLOSURES: The authors have no financial disclosures or conflicts of interest to declare.
10. Shnayder Y, Tsue TT, Toby EB, Werle AH, Girod DA. Safe osteocutaneous radial forearm flap harvest with prophylactic internal fixation. Craniomaxillofac Trauma Reconstr 2011;4:129-36.

11. Torina PJ, Matros E, Athanasian EA, Cordeiro PG. Immediate bone grafting and plating of the radial osteocutaneous free flap donor site. Ann Plast Surg 2013.

12. Nakamura T, Cooney WP III, Lui WH, et al. Radial styloidectomy: A biomechanical study on stability of the wrist joint. J Hand Surg Am 2001;26:85-93.

13. Weinzweig N, Jones NF, Shestak KC, Moon HK, Davies BW. Oromandibular reconstruction using a keel-shaped modification of the radial forearm osteocutaneous flap. Ann Plast Surg 1994;33:359-69.

14. Lutz BS, Ng SH, Cabailo R, Lin CH, Wei FC. Value of routine angiography before traumatic lower-limb reconstruction with microvascular free tissue transplantation. J Trauma 1998;44:682-6.

15. Militsakh ON, Wallace DI, Kriet JD, Tsue TT, Girod DA. The role of the osteocutaneous radial forearm free flap in the treatment of mandibular osteoradionecrosis. Otolaryngol Head Neck Surg 2005;133:80-3.

16. Day KE, Desmond R, Magnuson JS, Carroll WR, Rosenthal EL. Hardware removal after osseous free flap reconstruction. Otolaryngol Head Neck Surg 2014;150:40-6.

17. Lopez-Arcas JM, Arias J, Del Castillo JL, et al. The fibula osteomyocutaneous flap for mandible reconstruction: A 15-year experience. J Oral Maxillofac Surg 2010;68:2377-84.

18. Moscoso JF, Keller J, Genden E, et al. Vascularized bone flaps in oromandibular reconstruction. A comparative anatomic study of bone stock from various donor sites to assess suitability for enosseous dental implants. Arch Otolaryngol Head Neck Surg 1994;120:36-43. 
19. Thoma A, Khadaroo R, Grigenas O, et al. Oromandibular reconstruction with the radial-forearm osteocutaneous flap: Experience with 60 consecutive cases. Plast Reconstr Surg 1999;104:368-78.

20. Inglefield CJ, Kolhe PS. Fracture of the radial forearm osteocutaneous donor site. Ann Plast Surg 1994;33:638-42.

21. Vaughan ED. The radial forearm free flap in orofacial reconstruction. Personal experience in 120 consecutive cases. J Craniomaxillofac Surg 1990;18:2-7.

22. Bardsley AF, Soutar DS, Elliot D, Batchelor AG. Reducing morbidity in the radial forearm flap donor site. Plast Reconstr Surg 1990;86:287-92

23. Swanson E, Boyd JB, Mulholland RS. The radial forearm flap: A biomechanical study of the osteotomized radius. Plast Reconstr Surg 1990;85:267-72.

24. Meland NB, Maki S, Chao EY, Rademaker B. The radial forearm flap: A biomechanical study of donor-site morbidity utilizing sheep tibia. Plast Reconstr Surg 1992;90:763-73.

25. Waits CA, Toby EB, Girod DA, Tsue TT. Osteocutaneous radial forearm free flap: long-term radiographic evaluation of donor site morbidity after prophylactic plating of radius. J Reconstr Microsurg 2007;23:367-72.

26. Villaret DB, Futran NA. The indications and outcomes in the use of osteocutaneous radial forearm free flap. Head Neck 2003;25:475-81.

27. Werle AH, Tsue TT, Toby EB, Girod DA. Osteocutaneous radial forearm free flap: Its use without significant donor site morbidity. Otolaryngol Head Neck Surg 2000;123:711-7.

28. Yao J, Osterman AL. Arthroscopic techniques for wrist arthritis (radial styloidectomy and proximal pole hamate excisions). Hand Clin 2005;21:519-26.

29. Lorenzo JA, Raisz LG. Osteoporosis. In: Melmed S, Polonsky KS, Larsen PR, Kronenberg M, eds. Williams Textbook of Endocrinology. Philadelphia: Elsevier Publishing; 2011:1334.

30. Rockwell GM, Thoma A. Should the donor radius be plated prophylactically after harvest of a radial osteocutaneous flap? A cost-effectiveness analysis. J Reconstr Microsurg 2004;20:297-306. 